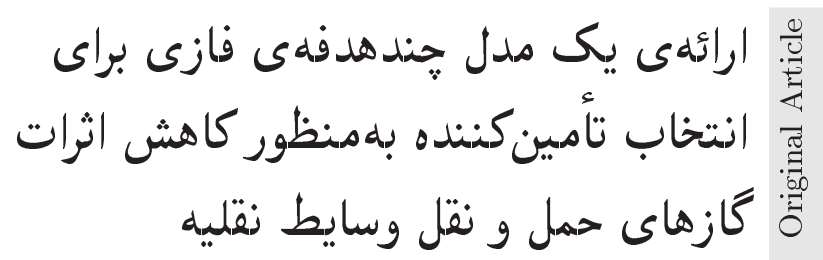

\author{
اكبر ميرزايى (كارشناس ارشد) \\ ابوالفضل كاظمى * (استاديار) \\ دانشكدهى مهندسى صنايع و مكانيك، دانشكاه آزاد اسلامى واحد قزوين
}

\section{r r بيشينهى تحقيق}

دحققين زيادى به مدلسازى و ارزيابى اجزاى دختلف دسئلهى انتخاب تأمينكننده

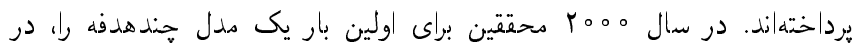

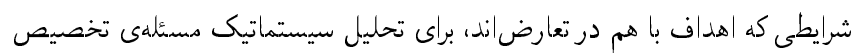

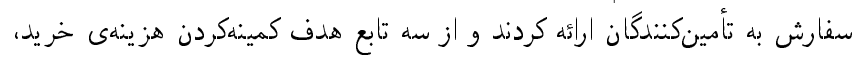

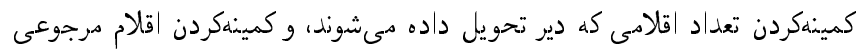

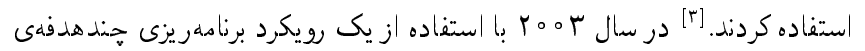

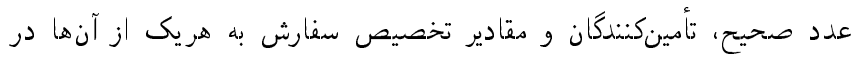

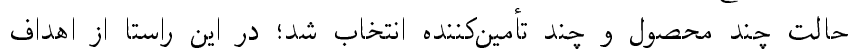

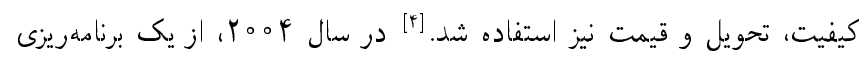

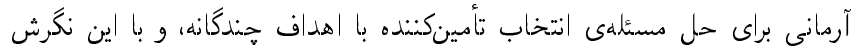

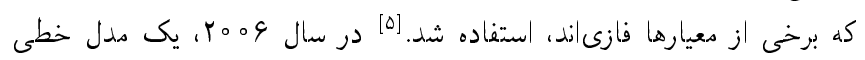

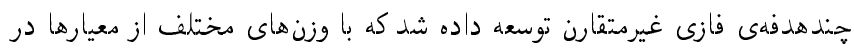

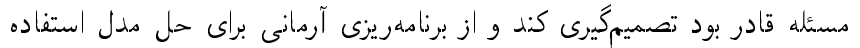

شد. شت] در سال 9 ه م ب، دحققين يك مدل جندهدفهى فازى وزندار افزايشى و متد

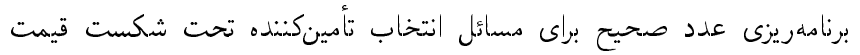

وازگًان كليدى: انتخاب تأمينكنده، بهينهسازى هندهدفه، آلايندهاى دحيط زيست، حمل و نقل وسايط نقليه، رويكرد فازى.

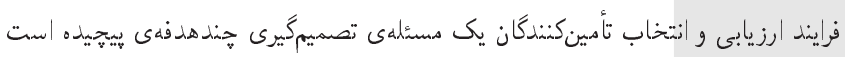

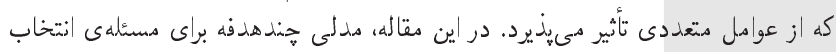

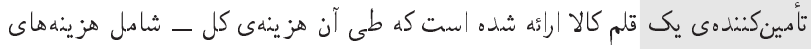

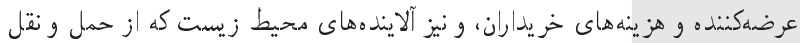

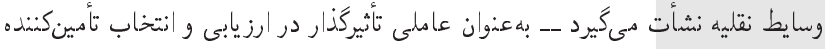

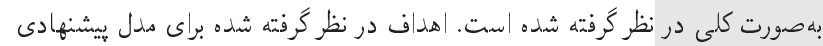

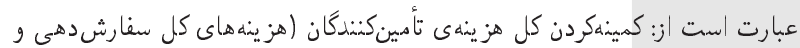

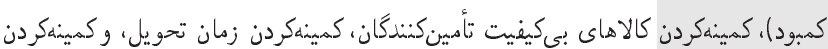

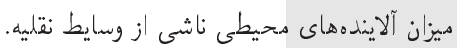

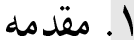

مسئلهى انتخاب تأمينكننده در زنجيرهى تأمين يك مسئلهى جديد نيست و ادبيات

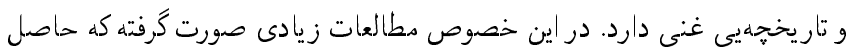

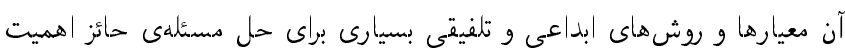

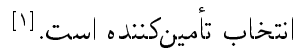

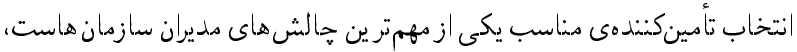
هرا كه تصميمات نادرست نه فقط خر يدار بلكه كل زنجيرهى تأمين را متأثر مى سازد.

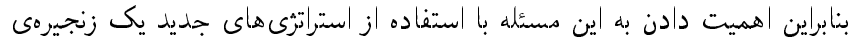

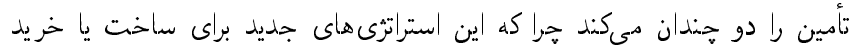

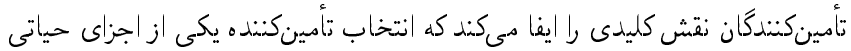

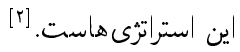

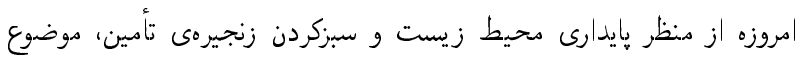

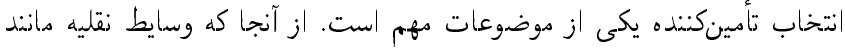

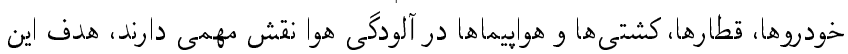

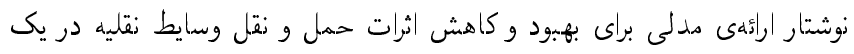

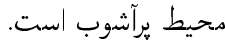

$$
\text { * تويسنده مسئول }
$$

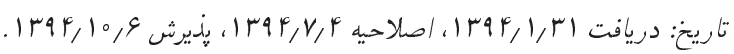


k: انديس توابع هدف

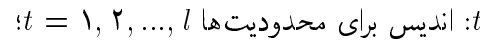

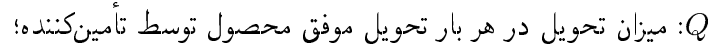
D

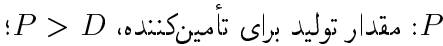
S $S_{V}$

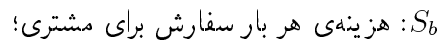
 h

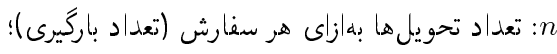

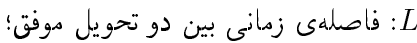

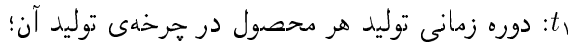
Tt T

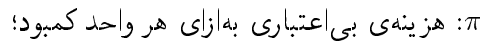

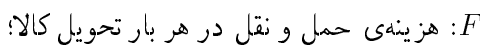
: d: هزينهى ترميم يا اصلاح هر دحصول خراب؛

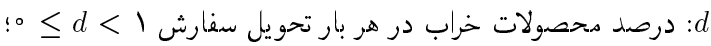

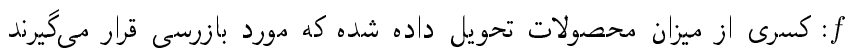
؛ $\leq f \leq 1$ : $C_{i}$ إن $C_{r}$ LOCi

در هر دوره؛

Mi CAPi C: $C_{m}$ : $U_{i}$ ه $(n, Q)$

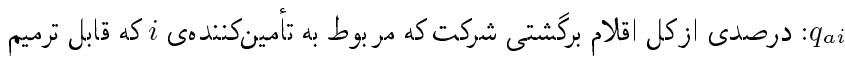
q q درصدى از كل اقلام برگشتى شركت كه مر بوط به تأمينكنندهى i كه قابل ترميم و اصلاح است؛ و اصلاح نيست؛

أثر Di

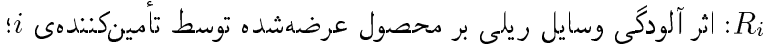
Zi Bi Im آ:

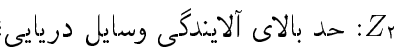

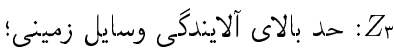

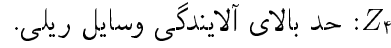

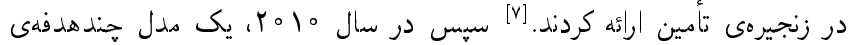

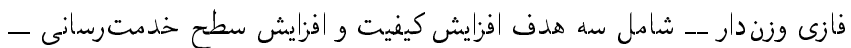

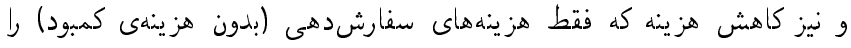

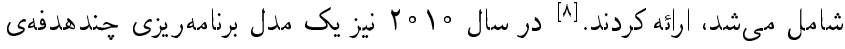

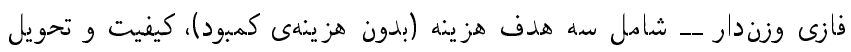

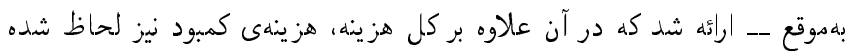

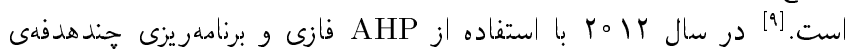

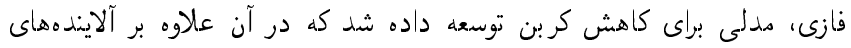

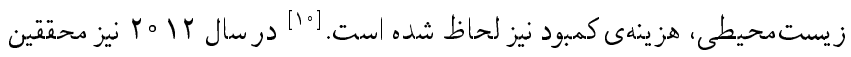

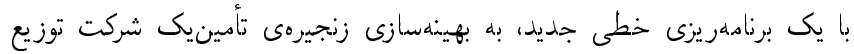

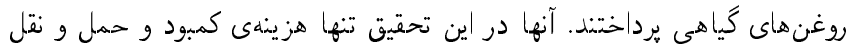

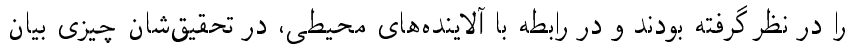
نشد.]'

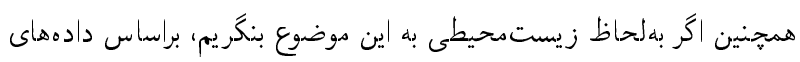

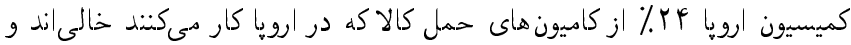

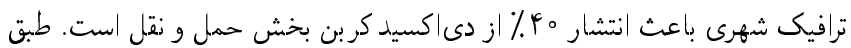

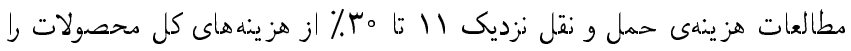

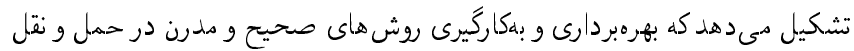

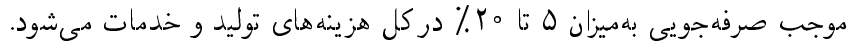
بنابراين كاهش حمل و نقل شهرى مىتواند باعث افزايش منافع اقتصادى و حفظ هن

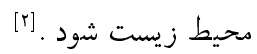
در تمامى تحقيقات كذشته، فقط در راستاى كاهش هز ينههاى زنجيرهى تأمين و

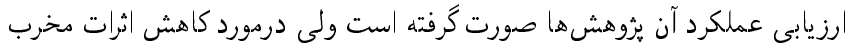

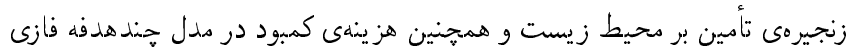

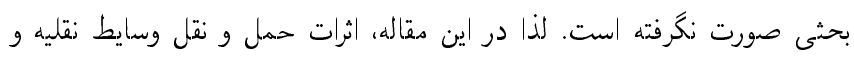

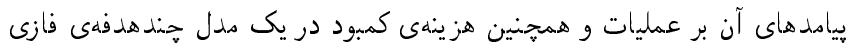
مورد دطالعه قرار داده شده است عديات همجن.

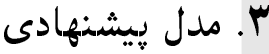

هدف اين مقاله ارائهى يك مدل رياضى هندهدفه فازى براى انتخاب تأمينكنده

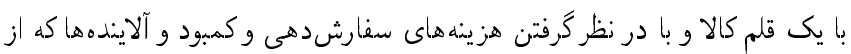

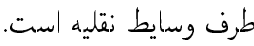

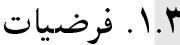

مفروضات هدل يشينهادى ذكر شده عبارت است از: تنها يك نوع دحصول

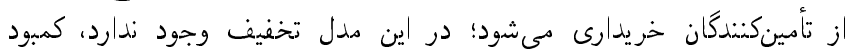

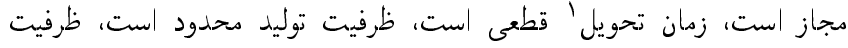

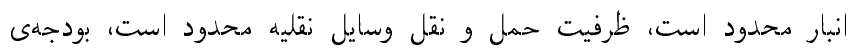

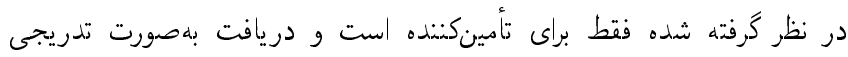




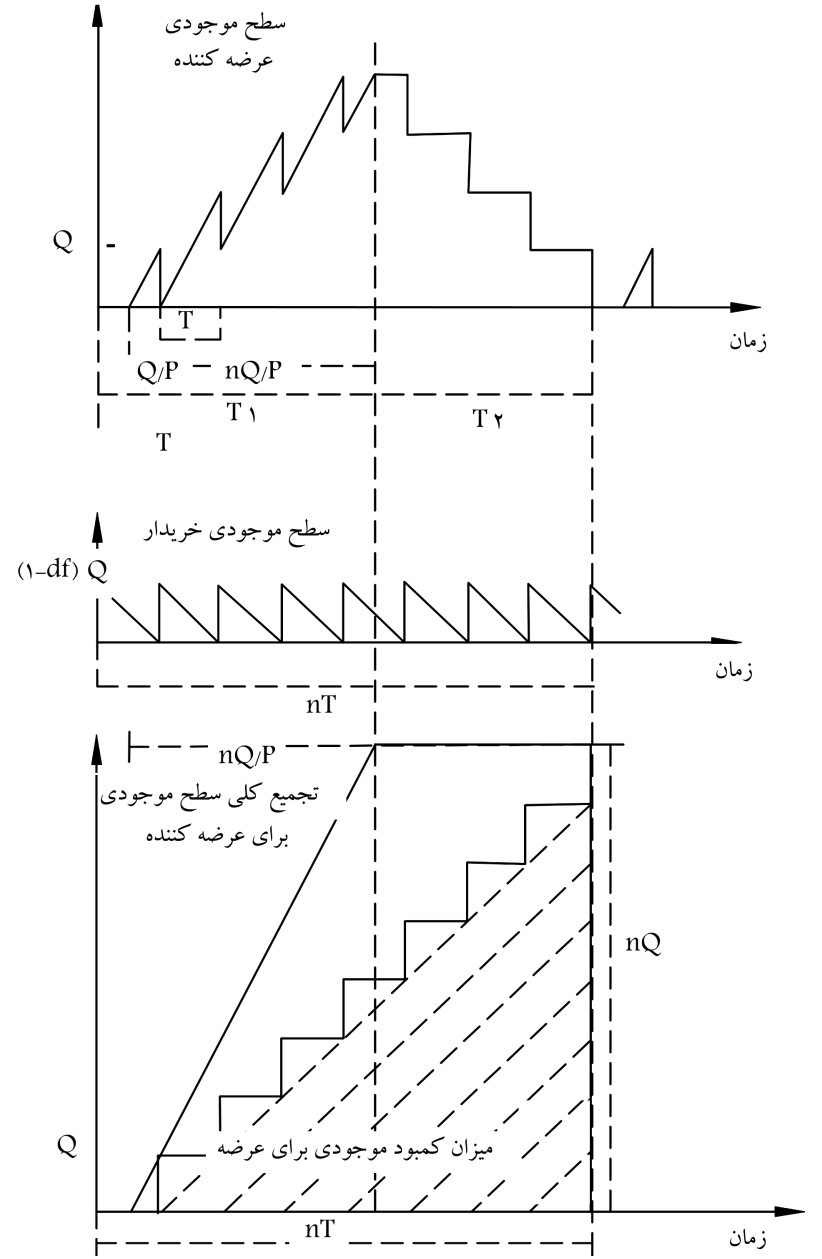

شكل 1. سطح موجودى براى تأمينكننده و خريدار و رفتار سيستم در طول زمان.

باكسرى از f محصولات را مورد بازرسى قرار مىدهد و به ميزان df از محتصولات

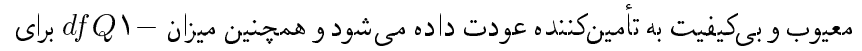

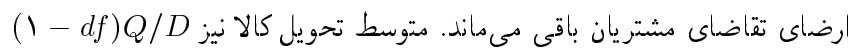

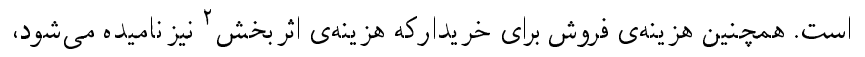

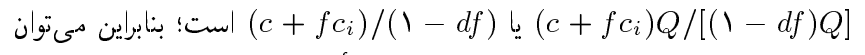

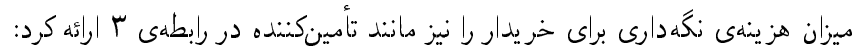

$H C_{B}(n, Q)=\left\{\frac{Q(1-d f)}{r} \times \frac{c+f c_{i}}{1-d f}\right\} h_{B}=\frac{Q\left(c+f c_{i}\right) h_{B}}{r}$

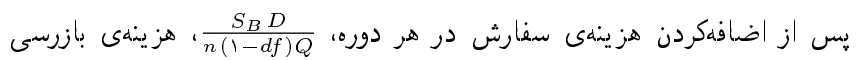

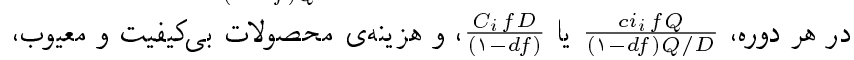
年

$$
\begin{aligned}
T C_{B}(n, Q) & =\frac{S_{B} D}{n(\backslash-d f) Q}+\frac{D\left(f c_{i}+d(\backslash-f) c_{r}\right)}{(1-d f)} \\
& +\frac{Q\left(C+f c_{i}\right)}{r} h_{b}
\end{aligned}
$$

روابط r و F هزينهى كل هر دوره خريدار و تأمينكنده (هزينهى كل يكيارجه) را
قرارداد)؛ مقدارتوليد/سفارش تخصيص داديم داده شده براى تأمينكندهى i در هر دوره (طبق

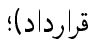

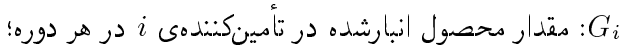
保:

\section{P.r. F. توابع هدف و محدوديتها}

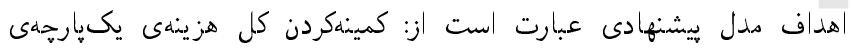

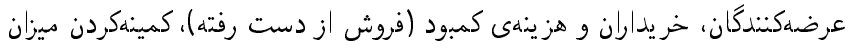

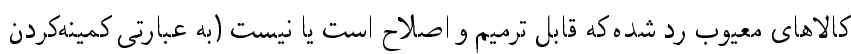

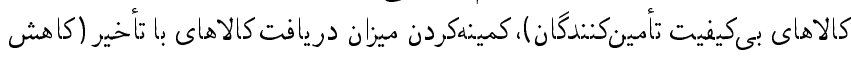

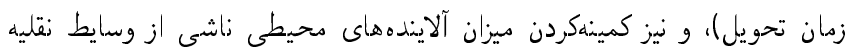

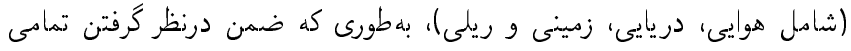

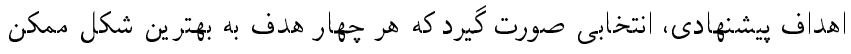

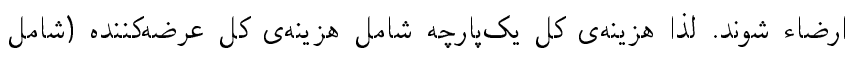

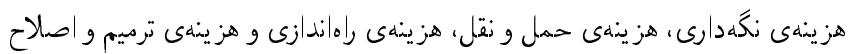

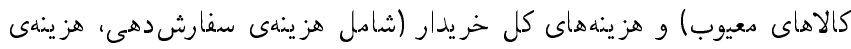

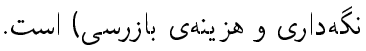

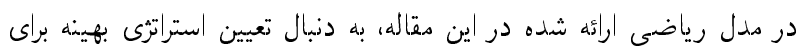

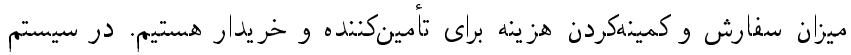

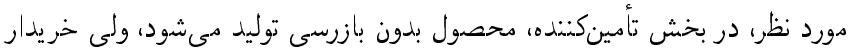

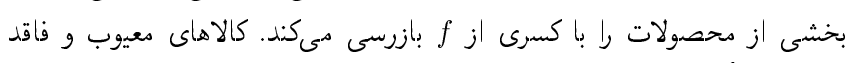

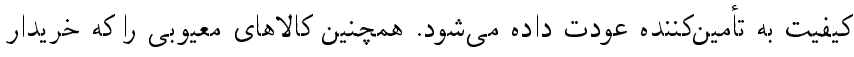

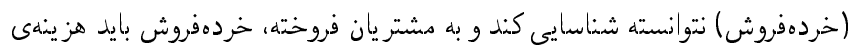

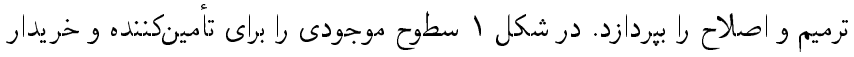

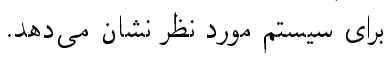

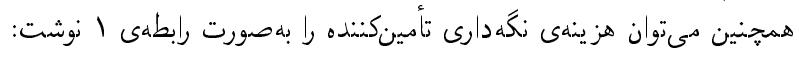

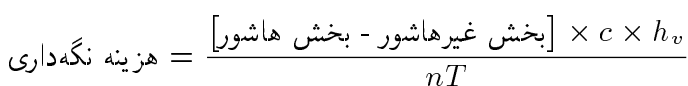
$\frac{\left\{\left\lfloor n Q\left(\frac{Q}{P}+(n-1) T-\frac{n Q\left(\frac{n Q}{P}\right)}{r}\right\rfloor-T[Q+r Q+L+(n-1) Q]\right\} c h_{v}\right.}{(n T)}$ $\frac{\left\{\left[n Q\left(\frac{Q}{P}+(n-1) \frac{(1-d f) Q}{D}\right)-\frac{n^{\curlyvee} Q^{\curlyvee}}{r P}\right]-\frac{(1-d f) Q}{D} \frac{(n-1) n Q}{r}\right\} c h_{v}}{\left(n \frac{(1-d f) Q}{D}\right)}$

$=\left\{\frac{Q}{r}+\frac{(n-r) Q}{r}\left(1-\frac{D}{(1-d f) P}\right)\right\} c h_{v}$

بس از اضافكردن هزينهى حمل و نقل، هزينهى راهاندازى و هزينهى ترميم و اصلاح محصولات معيوب، هزينهى كل تأمينكنده به دست مز مئي رآيد:

$$
\begin{aligned}
T C_{V}(n, Q)= & \frac{S_{V} D}{n(1-d f) Q}+\frac{F D}{(1-d f) Q}+\frac{v D d f}{(1-d f)} \\
& +\left\{\frac{Q}{r}+\frac{(n-r) Q}{r}\left(1-\frac{D}{(1-d f) p}\right)\right\} c h_{v}
\end{aligned}
$$

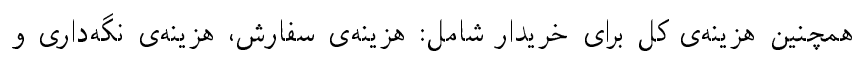

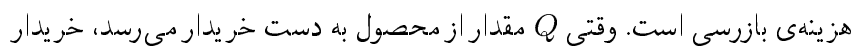


$\sum_{i=0}^{n} X_{i}=D$

$X_{i} \leq U_{i}$

$t_{1} X_{i} \leq L O G$,

$G_{i} M_{i} \leq C A P_{i}$

$\% 9 \circ X_{i} \leq G_{i} \leq X_{i}$

$Y_{i} \leq \sum C_{m} I_{m}$

$\% \vee X_{i} \leq Y_{i} \leq X_{i}$

$\sum_{i=0}^{n} H_{i} X_{i} \leq z 1$

$\sum_{i=\circ}^{n} D_{i} X_{i} \leq z r$

$\sum_{i=0}^{n} Z_{i} X_{i} \leq z r$

$\sum_{i=0}^{n} R_{i} X_{i} \leq z \uparrow$

$S_{i} X_{i} \leq B_{i}$

تابع هدف ^ كل هزينهى يكبارجه تأمينكندكان را كاهش مى مهدد. تابع هدف 9

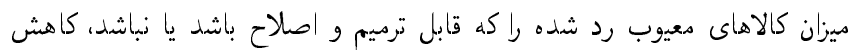

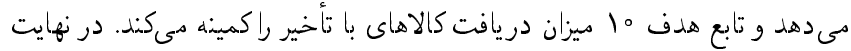

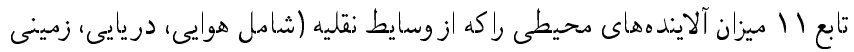

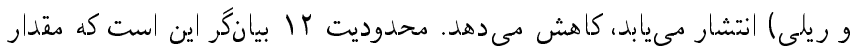

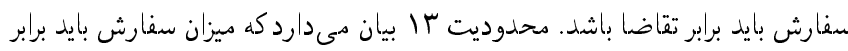

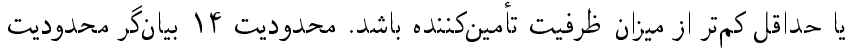

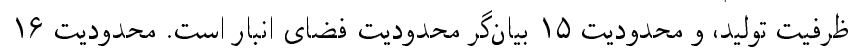

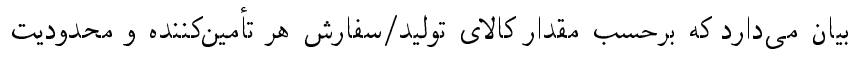

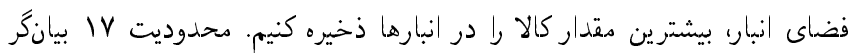

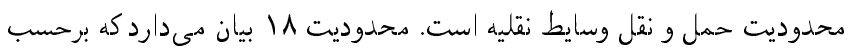

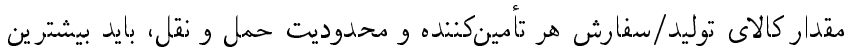

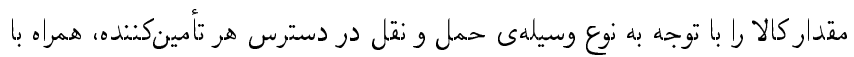

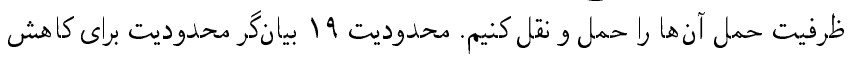

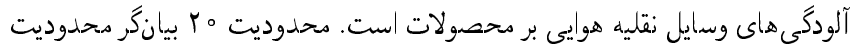

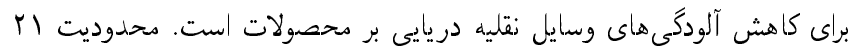

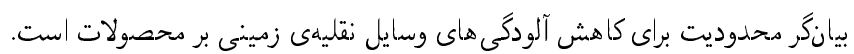

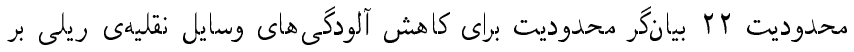

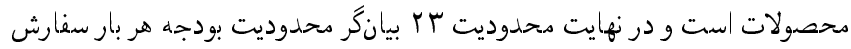

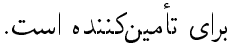

\section{|f أ. روشهاى حل بيشنهادى}

Zراين بخش بهنظور حل مدلهاى ارائه شده، ازرويكردهاى فازى Zimmermann و فازى جبرانى استفاده مى شود.

\section{Zimmermann 1. ا. رويكرد فازى}

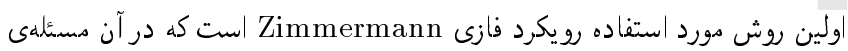

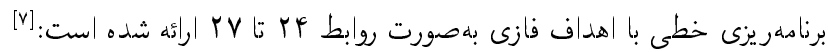

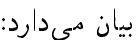

$$
K(n, Q)=T C_{V}(n, Q)+T C_{B}(n, Q)
$$

$$
\text { كه در آن بس از سادهازى، به رابطهى } 9 \text { دست مىيابيم: }
$$

$$
\begin{aligned}
K(n, Q)= & \frac{\left(S_{V}+S_{B}\right) D}{n(1-d f) Q}+\frac{F D}{(1-d f) Q} \\
& +\frac{D\left[v d f+f c_{i}+d(1-f) c_{r}\right]}{(1-d f) Q} \\
& +\left\{\frac{Q}{r}+\frac{(n-r) Q}{r}\left(1-\frac{D}{(1-d f) p}\right)\right\} c h_{v} \\
& +\frac{Q\left(c+f c_{r}\right)}{r} h_{B}
\end{aligned}
$$

در صورتى كه هزينهى كمبود را نيز اضافه كنيم (شكل r)، مدل نهايى بهصورت

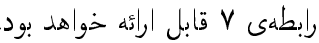

$$
\begin{aligned}
K(n, Q)= & \frac{\left(S_{V}+S_{B}\right) D}{n(1-d f) Q}+\frac{F D}{(1-d f) Q} \\
& +\frac{D\left[v d f+f c_{i}+d(1-f) c_{r}\right]}{(1-d f) Q} \\
& +\left\{\frac{Q}{r}+\frac{(n-r) Q}{r}\left(1-\frac{D}{(1-d f) p}\right)\right\} c h_{v} \\
& +\frac{Q\left(c+f c_{r}\right)}{r} h_{B}+\frac{1}{r} \pi D\left(T-t_{1}\right)^{r}
\end{aligned}
$$

بنابراين، مدل كلى بهصورت رابطههاى 1 تا آץ ارائه مىشود:

$\min z \backslash=\sum_{1}^{n} \cdot\left(\begin{array}{l}K(n, Q)=\frac{\left(S_{v}=S_{B}\right) D}{n(1-d f) Q}+\frac{F D}{(1-d f) Q} \\ +\frac{\left.D\left[v d f+f c_{1}+d()-f\right) c_{r}\right]}{(-d f)} \\ +\left\{\frac{Q}{r}+\frac{(n-r) Q}{r}\left(1-\frac{D}{(1-d f) P}\right)\right\} c h_{v} \\ +\frac{Q\left(c+f c_{1}\right)}{r} h_{B}+\frac{1}{r} \pi D\left(T-t_{1}\right)^{r}\end{array}\right) x_{i}$

$\operatorname{Min} x \boldsymbol{r}=\sum_{i=。}^{n} q_{i a} X_{i}+\sum_{i=。}^{n} q_{i b} X_{i}$

$\operatorname{Min} z r=\sum_{i=。}^{n} L_{i} X_{i}$

$\operatorname{Min} z \boldsymbol{f}=\sum_{i=\circ}^{n} H_{i} X_{i}+\sum_{i=。}^{n} D_{i} X_{i}$

$$
+\sum_{i=0}^{n} Z_{i} X_{i} \sum_{i=。}^{n} R_{i} X_{i}
$$

S.t :

سطح موجودى (t) (

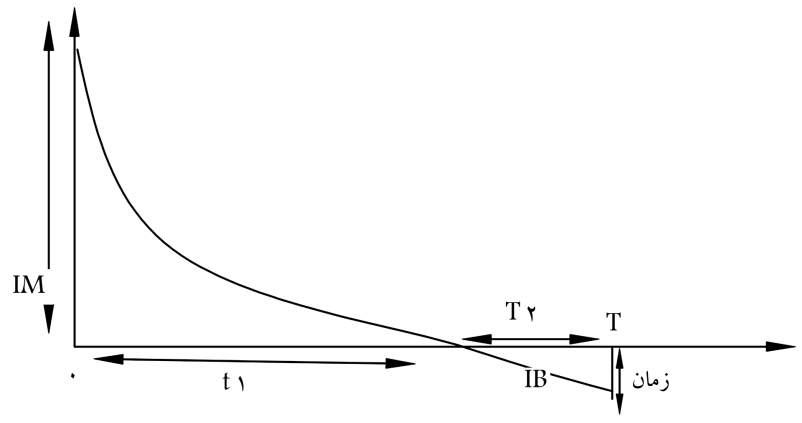

شكل r. سطح موجودى براى تأمينكننده با وجود كمبود. 


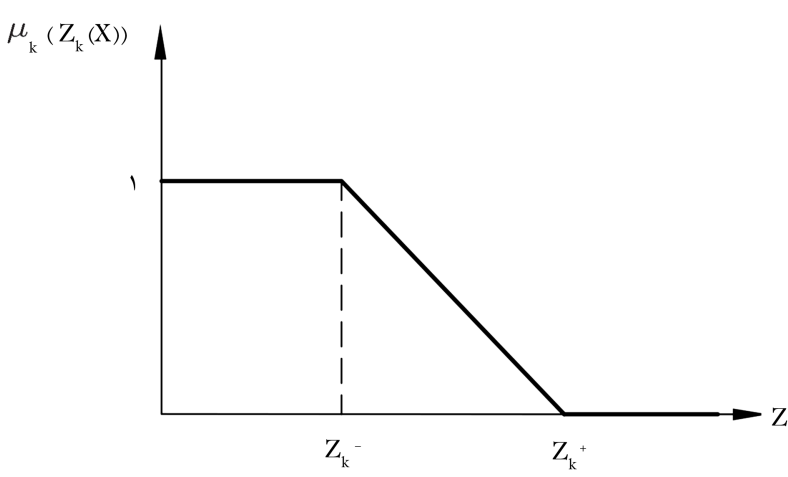

شكل r. تابع عضويت خطى براى توابع هدف از نوع منفى.

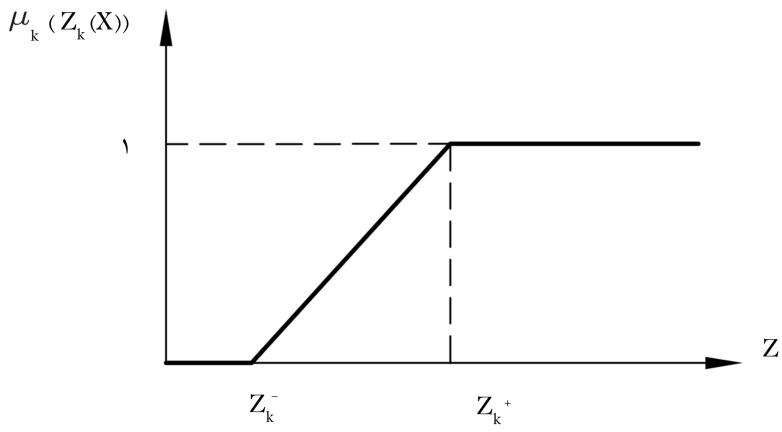

شكل f. تابع عضويت خطى براى توابع هدف از نوع مثبت.

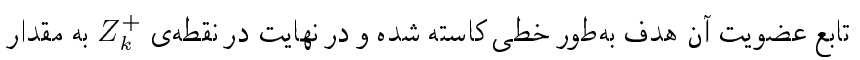

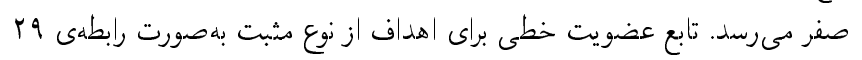
تعريف شده است

$$
\begin{aligned}
& \mu_{k}\left(Z_{k}(x)\right)= \\
& \begin{cases}1 & \text { for } Z_{k}(x) \leq Z_{k}^{-} \\
\frac{\left(Z_{k}(x)-Z_{k}^{-}\right)}{\left(Z_{k}^{+}-Z_{k}^{-}\right)} & \text {for } Z_{k}^{-}<Z_{k}(x)<Z_{k}^{+} \quad k=(\mathrm{I}, \mathrm{r}, \ldots, q) \\
0 & \text { for } Z_{k}(x) \geq Z_{k}^{-}\end{cases}
\end{aligned}
$$

همخنين تابع عضويت خطى (x) نشان داده شده است.

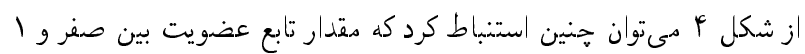

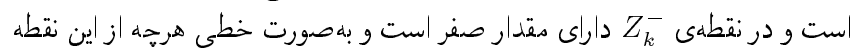

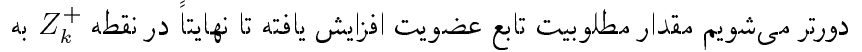

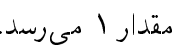

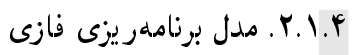

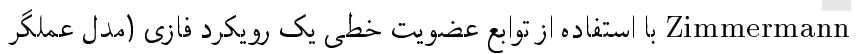

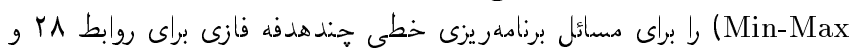

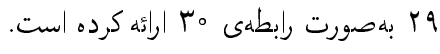

$\max _{x} \min _{k} \mu_{k}\left(Z_{k}(x)\right)$

S.t $\quad x \in x_{d}$
$\tilde{Z}_{k}=\sum_{i=1}^{n} c_{k i} x_{i} \leq \approx Z_{k}^{\circ}=1, \Gamma, \ldots, q \quad$ (براى توابع از نوع منفى)

$\tilde{Z}_{k}=\sum_{i=1}^{n} c_{k i} x_{i} \geq \approx Z_{k}^{\circ}=1, r \ldots, q \quad$ (براى توابع از نوع مثبت)

S.t.

$g_{t}(x)=\sum_{i=1}^{n} a_{t i} x_{i} \leq b_{t} \quad t=1, r, \ldots ., l$

$x_{i} \geq \circ \quad i=1, r, \ldots, n$

جايى كه

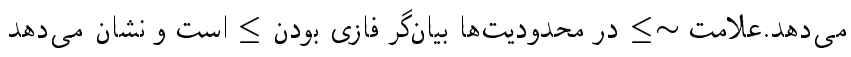

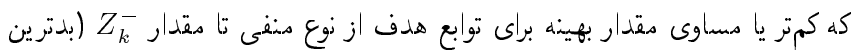

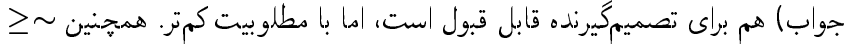

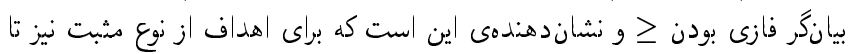

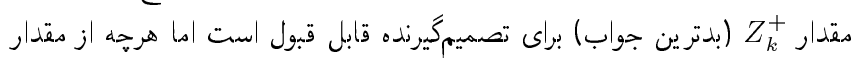

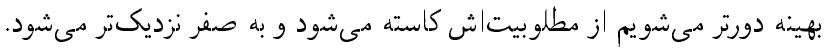

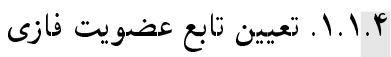

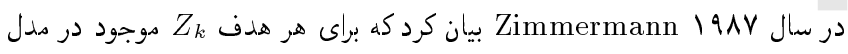

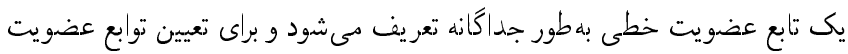

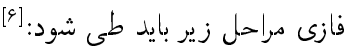

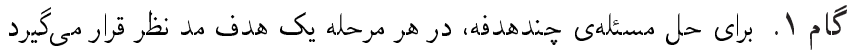

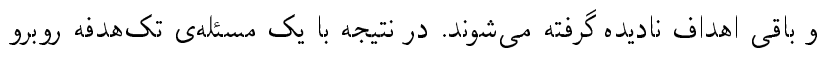

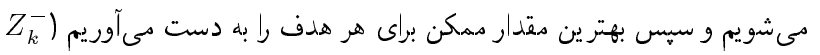

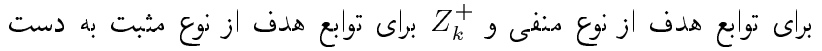

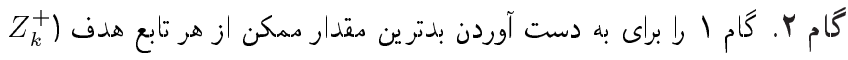

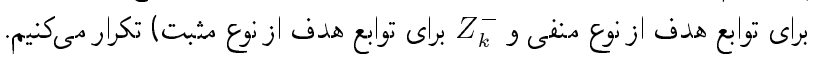

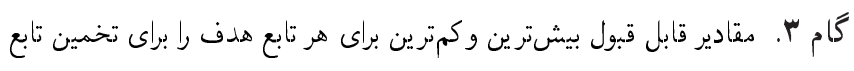
عضويت هر هدف استفاده مىكنيم.

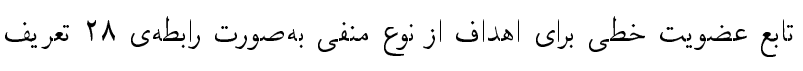

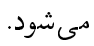

$\mu_{k}\left(Z_{k}(x)\right)=$

$$
\begin{cases}1 & \text { for } Z_{k}(x) \leq Z_{k}^{-} \\ \frac{\left(Z_{k}^{+}-Z_{k}(x)\right)}{\left(Z_{k}^{+}-Z_{k}^{-}\right)} & \text {for } Z_{k}^{-}<Z_{k}(x)<Z_{k}^{+} \quad k=(1, r, \ldots, q) \\ 0 & \text { for } Z_{k}(x) \geq Z_{k}^{+}\end{cases}
$$

همنين تابع عضويت خطى (x) نشان داده شده است.

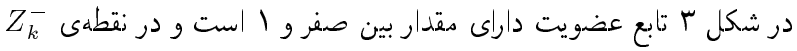

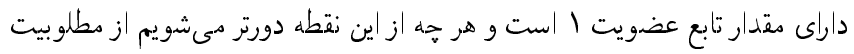


بنابراين، مدل جبرانى ذكر شده در بالا راه حلهاى به شدت كاراى جبرانى را براى

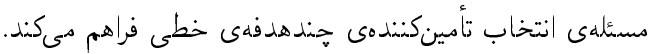

\section{צ. حل مثال عددى با رويكردهاى برنامهريزى رياضى و}

فازى

در اين مثال خر يدار نياز به خريد يك قلم كالا از بهتر ين تأمينكنندكان و تخصيص

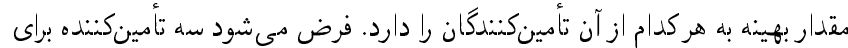

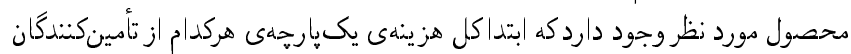

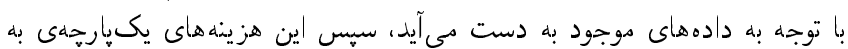

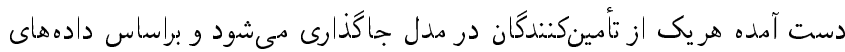

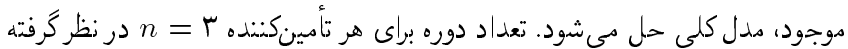

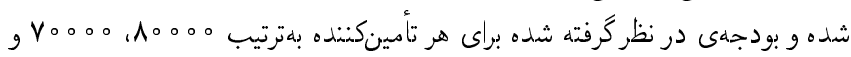

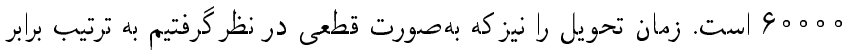

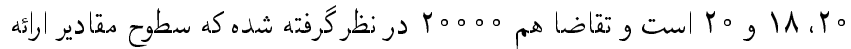

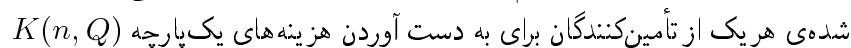
در جدول ال ارائه شده است.

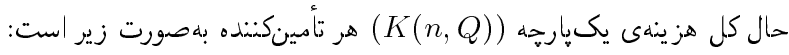

$$
\begin{aligned}
& K_{1}(N=r, Q)=\frac{(r \Delta+r) \times r 0000}{r(1-0,0 \times 0,9 \Delta) \times \Delta \Delta 00} \\
& +\frac{(1-0,01 \times 0,90) \times \Delta 000}{\left(k \times r_{0} \times 00\right)} \\
& +\frac{r 000[9 \times 0,0 r \times 0,90 \times r+0,0 Y(1-0,90) \times V]}{(1-0,0 Y \times 0,90)} \\
& +\left\{\frac{\Delta \Delta 00}{r}+\frac{(r-r) \times \Delta \Delta 00}{r}\right.
\end{aligned}
$$

\begin{tabular}{|c|c|c|c|}
\hline$r$ & $r$ & 1 & تأمينكنندگًان \\
\hline$V Y$ & $v_{0}$ & $\mathrm{VA}$ & $C$ \\
\hline rr & ro & ro & $S_{V}$ \\
\hline r & q & $r$ & $S_{b}$ \\
\hline 0 & $r$ & 0 & $h_{v}$ \\
\hline$r$ & $r$ & $r$ & $h_{b}$ \\
\hline$r$ & r & r & $f$ \\
\hline 9 & v & 9 & $V$ \\
\hline$r$ & $\varphi$ & r & $C_{i}$ \\
\hline v & $\wedge$ & v & $C_{r}$ \\
\hline io & rı & ro & $\pi$ \\
\hline$\wedge$ min & $\wedge$ min & $10 \min$ & $t_{1}$ \\
\hline romin & $1 \wedge \min$ & romin & $T$ \\
\hline $0,0 \mathrm{r}$ & 0,00 & $0,0 r$ & $d$ \\
\hline 0,9 & 0,94 & 0,90 & $f$ \\
\hline 0000 & 9000 & $\Delta \Delta 0^{\circ}$ & $Q$ \\
\hline vroo & voo. & v10。 & $P$ \\
\hline
\end{tabular}

جدول ا. سطوح مقادير هريك از تأمينكندانان براى به دست أوردن هزينههاى يكيارجه
معادلهى مب با استفاده از تعريف يك متغير كمكى ل به رابطهى آ تبديل مى شود: $\lambda \max$

S.t.

$\mu_{k}\left(Z_{k}(x)\right) \geq \lambda, k=(1, r, \ldots, q)$

$x \in x_{d} \quad \circ \leq \lambda \leq 1$

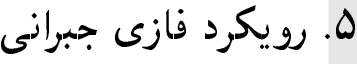

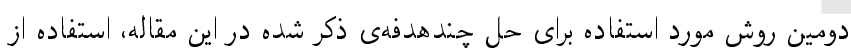

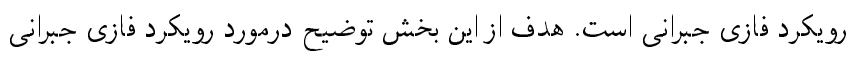

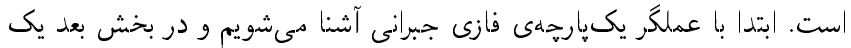

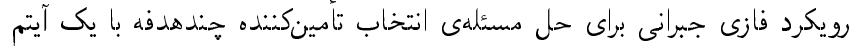
ارائه شده است.

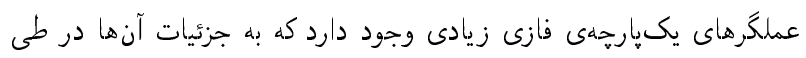

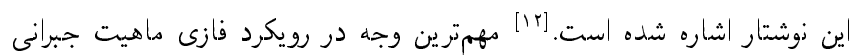

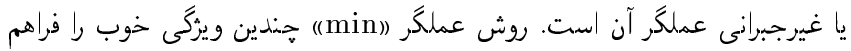

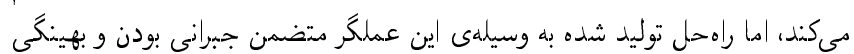

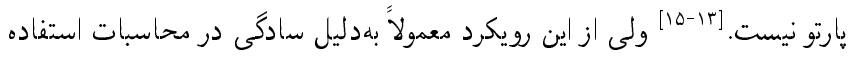

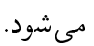

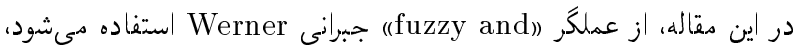

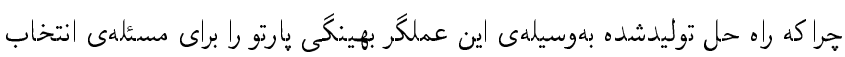

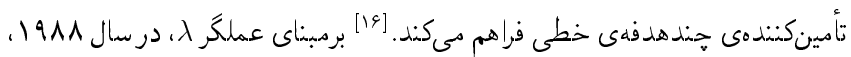

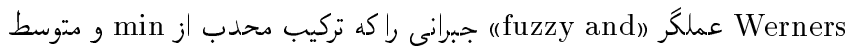

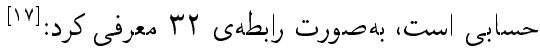

$\mu_{\text {and }}=\gamma \min _{k}\left(\mu_{k}\right) \frac{(1-\gamma)}{m}\left(\sum \mu_{k}\right)$

كه مى كند. با قرار دادن مى يابد.

هـ ا. يكى رويكرد فازى جبرانى براى مسئلهى انتخاب تأمينكننده

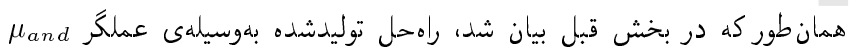

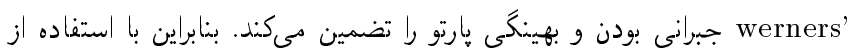
عملرً werners' $\mu_{\text {and }}$ Max $\mu_{\text {and }}=\lambda+\frac{(1-\gamma)}{k} \sum_{k=1}^{q} \lambda_{k}$ s.t : $x \in X_{k}$ $\mu_{k}\left(Z_{k}(x)\right) \geq \lambda+\lambda_{k}$

$\lambda+\lambda_{k} \leq 1$

$\lambda, \psi \lambda_{k} \in\left[{ }^{\circ}, 1\right], \quad k=1, r, \ldots, q$

$\gamma \in\left[{ }^{\circ}, 1\right]$ 


$$
\begin{aligned}
& 0,1 \mu r \times X_{r}<=V F_{0} \circ, \\
& \circ, 10 \times X_{r}<=\vee v \circ \circ \\
& \circ, \Delta \times G,<=\vee \Delta \circ \circ \circ, \\
& \circ, 9 \vee \times X_{1}<=G_{1}, \\
& G_{1}<=X_{1} \text {, } \\
& \circ, \boldsymbol{F} \times G_{1}<=r r_{\circ} \circ, \\
& \circ, 9 \vee \times X_{r}<=G_{r}, \\
& G_{r}<=X_{r} \text {, } \\
& \circ, 9 \times G,<=00 \circ \circ, \\
& \circ, 9 \vee \times X_{r}<=G_{r} ; \\
& G_{r}<=X_{r} \text {; } \\
& Y<=100 \circ \circ \\
& \circ \wedge \times x_{\curlywedge}<=y_{\curlywedge} ; \\
& y_{1}<=x_{\curlywedge} \text {; } \\
& Y_{r}<=11000 ; \\
& \circ, \wedge \times x_{r}<=y_{r} ; \\
& y_{r}<=x_{r} ; \\
& Y_{r}<=900 \circ ; \\
& \circ, \wedge \times x_{r}<=y_{r} ;
\end{aligned}
$$

\begin{tabular}{|c|c|c|c|c|}
\hline$Z_{\varphi}$ & $Z_{\mu}$ & $Z_{Y}$ & $Z_{1}$ & \\
\hline$\Delta \circ r \Delta \circ$ & rqoroo & $19 \Delta F_{0}$ & 1098490 & $Z_{k}^{+}$ \\
\hline YAOV。 & rAvoo。 & $190 r 4$ & 10.0100 & $Z_{k}^{-}$ \\
\hline
\end{tabular}$$
y_{r}<=x_{r}
$$$$
0, \Lambda \times x_{1}+0, \mu \times x_{r}+0,9 \times x_{r}<=\mu_{0 \circ 0 \circ ;}
$$$$
0, r \times x_{1}+0, r \times x_{r}+0,9 \times x_{r}<=10000 ;
$$$$
0,9 \times x_{1}+1 \times x_{r}+0, \wedge \times x_{r}<=\mu_{0 \circ 0 \circ ;}
$$$$
0, \boldsymbol{r} \times x_{1}+0, q \times x_{r}+0, \nabla \times x_{r}<=r \Delta 00 \circ ;
$$$$
X_{1}<=\wedge \circ \circ \circ \circ
$$$$
X_{Y}<=\vee \circ \circ \circ ;
$$$$
X_{r}<=9000 \circ
$$$$
X_{i} \geq \circ, G_{i} \geq \circ, Y_{i} \geq \circ
$$$$
i=1, r, r, \quad m=1, r, r, r
$$

از حل تك تك اهداف و با كليهى دحدوديتهاى مدل با استفاده از نرمافزار GAMS

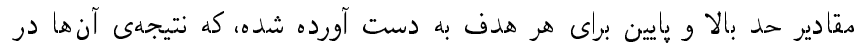
جدول r ارائه شده است.

جدول r. مقادير حد بالا و يايين براى هر هدف.

\begin{tabular}{|c|c|c|c|}
\hline$r$ & $r$ & 1 & تأمينكنندكان \\
\hline$v 0,0$ & $q r, r$ & $v 0, v$ & $(n, q) k$ \\
\hline 0,90 & $0,1 \Delta$ & 0,9 & Qia \\
\hline 0,01 & 0,1 & 0,00 & $Q i b$ \\
\hline 1,0 & $1, v$ & $1, r$ & $H_{i}$ \\
\hline $0, r$ & 0,0 & 0,1 & Di \\
\hline$r, r$ & $r, \Lambda$ & $r, 0$ & $Z_{i}$ \\
\hline $1, r$ & 1,1 & $1, r$ & $R_{i}$ \\
\hline 40000 & Voooo & YYOOO & $B_{i}$ \\
\hline Voo o & $1 F 000$ & 9000 & $U_{i}$ \\
\hline$r \min$ & $\Delta \min$ & $i \min$ & $L O G_{i}$ \\
\hline 9000 & 9000 & 9000 & $C A P_{i}$ \\
\hline 100 & 100 & 100 & $C_{m}$ \\
\hline 9 & 0 & v & $I_{m}$ \\
\hline
\end{tabular}

$$
\begin{aligned}
& \left.\left(1 \frac{(1-0,04 \times 0,90) \times R / 0^{\circ}}{1,4000}\right)\right\} \times V \wedge \times 0 \\
& +\frac{\Delta \Delta \circ 0(V \Lambda+0,9 \Delta \times r)}{r} \times r+\frac{1}{r} \times r \Delta
\end{aligned}
$$

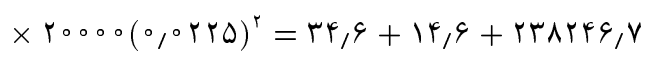

$$
\begin{aligned}
& -q 1 r 1 \circ V+99 \vee \circ 1 r, 0+V \Lambda v=V \Delta, V
\end{aligned}
$$

$K_{r}(N=r, Q)=9 r, r$

$K_{r}(N=r, Q)=\vee \circ, 0$

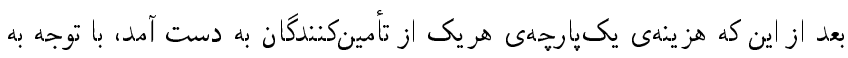

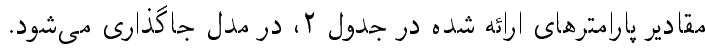

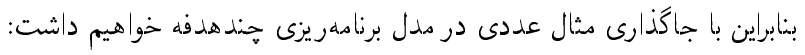

Objective function :

$\operatorname{Min} z l=\vee \Delta, \mathrm{V} \times X_{1}+q \mathrm{r}, \mathrm{r} \times X_{r}+\vee_{0}, \Delta \times X_{r} ;$

Min $z r=\left(00,9 \times X_{1}+0, \wedge \Delta \times X_{r}+0,9 \Delta \times X_{r}\right)$

$$
+\left(0,01 \times X_{1}+0,1 \times X_{r}+0,0 \wedge \times X_{r}\right)
$$

Min $z r=r \circ \times x_{\uparrow}+1 \Lambda \times x_{r}+r \circ x_{r} ;$

Min $z^{\boldsymbol{F}}=\left(0, \Lambda \times x_{\uparrow}+0,0 \times x_{\uparrow}+0, \boldsymbol{\varphi} \times x_{r}\right)$

$$
\begin{aligned}
& +\left(0, r \times x_{\Lambda}+0, \mu \times x_{r}+0,9 \times x_{r}\right) \\
& +\left(0,9 \times x_{\curlywedge}+1 \times x_{r}+0, \wedge \times x_{r}\right) \\
& +\left(0, \uparrow \times x_{\Lambda}+0,9 \times x_{r}+0, \nabla \times x_{r}\right)
\end{aligned}
$$

S.t :

$$
\begin{aligned}
& X_{1}+X_{r}+X_{r}=\text { r०००。; } \\
& X_{1}<=\wedge \vee \circ \circ ; \\
& X_{r}<=90 \circ \circ \text {; } \\
& X_{r}<=90 \circ \circ ; \\
& \circ 10 \times X_{1}<=\wedge \circ \circ \circ
\end{aligned}
$$


$\operatorname{Max} \mu_{\text {and }}=\lambda+\frac{(1-\gamma)}{r}\left(\lambda_{1}+\lambda_{r}+\lambda_{r}+\lambda_{r}\right)$

s.t :

$\mu_{1}\left(Z_{1}(x)\right)=\left(\frac{1099490-Z_{1}}{9111 \% \circ}\right) \geq \lambda+\lambda_{1}$

$\mu_{r}\left(Z_{r}(x)\right)=\left(\frac{19 \Delta Y^{\circ}-Z_{r}}{\Delta \circ \mathcal{F}}\right) \geq \lambda+\lambda_{r}$

$\mu_{r}\left(Z_{\digamma}(x)\right)=\left(\frac{r q \Delta \digamma_{\circ} \circ-Z_{r}}{\Lambda \digamma_{\circ} \circ}\right) \geq \lambda+\lambda_{r}$

$\mu_{\psi}\left(Z_{\psi}(x)\right)=\left(\frac{\Delta \circ r \Delta^{\circ}-Z_{\digamma}}{19 \Lambda^{\circ}}\right) \geq \lambda+\lambda_{\digamma}$

$\lambda+\lambda_{1} \leq 1$

$\lambda+\lambda_{r} \leq 1$

$\lambda+\lambda_{r} \leq 1$

$\lambda+\lambda_{r} \leq 1$

$\lambda, \lambda_{1}, \lambda_{r}, \lambda_{r}, \lambda_{r} \geq \circ \quad k=1, r, \ldots, q$

where $\gamma \in\left[{ }^{\circ}, 1\right]$

$x \in X_{d}, X_{d}=\left\{x / g(x) \leq b_{r}, r=1, r, \ldots, m\right\}$

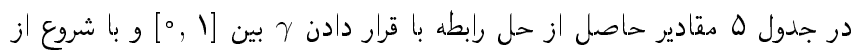

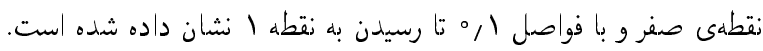

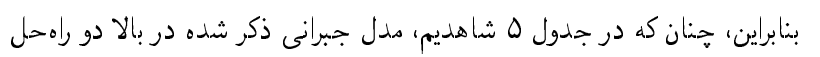

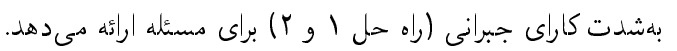

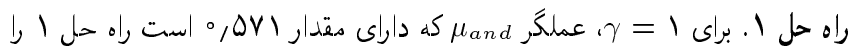

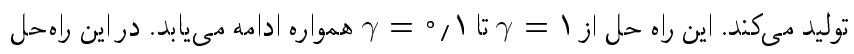

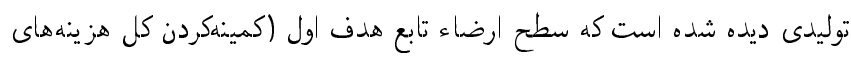

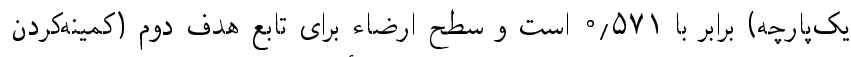

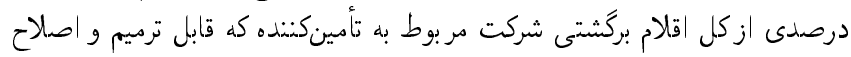

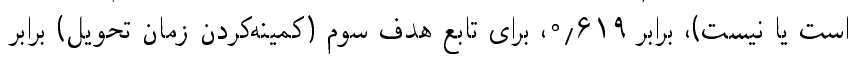

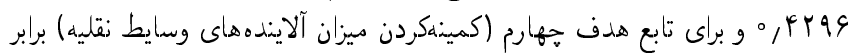

است

راهحل r. دومين راهحل توليد شده مريوط به هاته

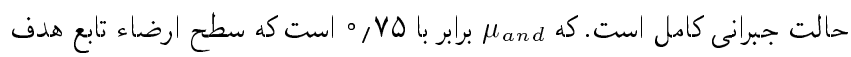

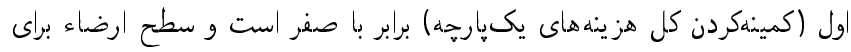

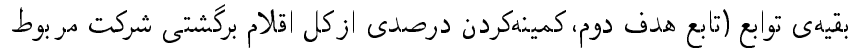

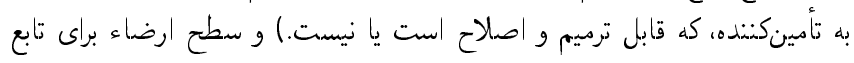

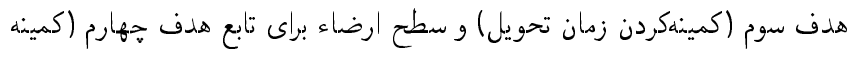

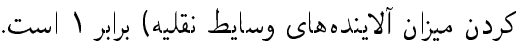

\section{צ.r. مقايسهى رويكرد فازى Zimmermann و رويكرد فازى}

جبرانى

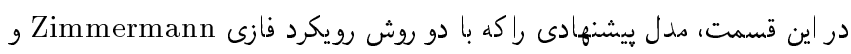

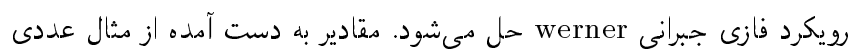

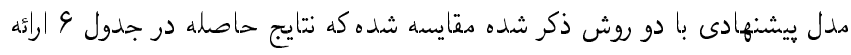

Zimmermann

مقادير تابع عضويت براى هريك از اهداف كه در جدول r به دست آمده، در مدل

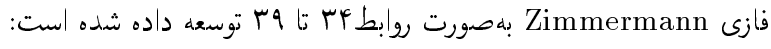

$\operatorname{Max} \lambda$

S.t.

$\lambda_{1} \leq\left(\frac{1099490-Z_{1}}{9111 \% \circ}\right)$

$\lambda_{r} \leq\left(\frac{19 \Delta \mu_{\circ}-Z_{r}}{\Delta \circ \varphi^{F}}\right)$

$\lambda_{r} \leq\left(\frac{\mu q \Delta F_{0} \circ-Z_{r}}{1 F_{\circ} \circ}\right)$

$\lambda_{\psi} \leq\left(\frac{\Delta \circ r \Delta \circ-Z_{\psi}}{19 \Lambda_{0}}\right)$

$x \in X_{d}, \quad X_{d}=\left\{x / g(x) \leq b_{r}, r=1, r, \ldots, m\right\}$

كه نتيجهى حل مدل فازى Zimmermann در جدول f ا ارائه شده است.

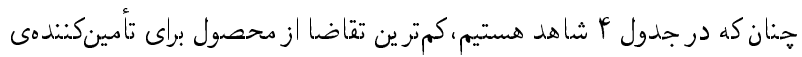

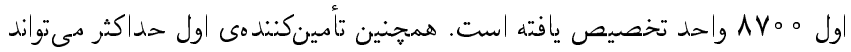
از

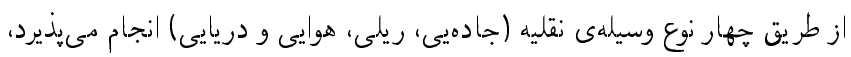

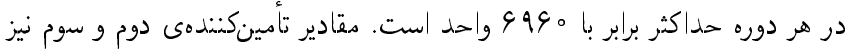
به همين ترتيب در جدول F أرائه شده است.

צ.r. حل مدل ارائه شده با استفاده از رويكرد فازى جبرانى

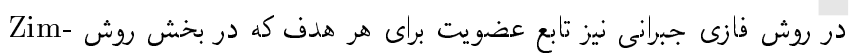

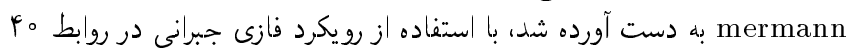
تا ملا نوشته شده است.

جدول F. نتايج حاصل از حل مدل با استفاده از رويكرد فازى Zimmermann.

\begin{tabular}{|c|c|c|c|}
\hline مقادير يارامترها & يارامترها & مقادير يارامترها & يارامترها \\
\hline ravv & $G_{r}$ & $0, \Delta V I F r$ & $\lambda$ \\
\hline 9914 & $G_{r}$ & IOFFTIO & $Z_{1}^{*}$ \\
\hline 9990 & $Y_{1}$ & I ITrA & $Z_{r}^{*}$ \\
\hline rrA。 & $Y_{r}$ & rq110。 & $Z_{r}^{*}$ \\
\hline$\Delta V \varphi_{0}$ & $Y_{r}$ & pqrqo & $Z_{\varphi}^{*}$ \\
\hline 0,0909 & $\mu_{\backslash} Z_{\backslash}(x)$ & $\Lambda V_{0} \circ$ & $X_{1}$ \\
\hline 0,9191 & $\mu_{\curlyvee} Z_{\Upsilon}(x)$ & 4100 & $X_{r}$ \\
\hline 0, GYAG & $\mu_{\digamma} Z_{\Upsilon}(x)$ & VYoo & $X_{r}$ \\
\hline $0, \Delta V I F$ & $\mu_{\uparrow} Z_{\uparrow}(x)$ & AFra & $G_{1}$ \\
\hline
\end{tabular}


جدول ه. نتايج حاصل از حل مدل با استفاده از رويكرد فازى جبرانى.

\begin{tabular}{|c|c|c|c|c|c|c|c|c|c|c|}
\hline & $\gamma=1$ & $\gamma=0,9$ & $\gamma=0, \wedge$ & $\gamma=0, \vee$ & $\gamma=0,4$ & $\gamma=0, \Delta$ & $\gamma=0, f$ & $\gamma=0, \mu$ & $\gamma=0, r$ & $\gamma=0,1$ \\
\hline$X_{1}$ & Avo。 & $\Lambda V_{0} \circ$ & $\Lambda V_{0} \circ$ & $\Lambda V_{0} \circ$ & $\Lambda V_{0} \circ$ & Avo。 & $\wedge V_{0} \circ$ & $\wedge V_{0} \circ$ & $\Lambda V_{0} \circ$ & $\Lambda V_{0} \circ$ \\
\hline$X_{r}$ & 4100 & 4100 & 4100 & 4100 & 4100 & 4100 & 4100 & 4100 & 9000 & 9000 \\
\hline$X_{r}$ & VYoo & VYoo & VYoo & VYoo & VYoo & VYoo & VYoo & VYoo & $\varphi \Lambda_{0} \circ$ & r $\Lambda_{0} \circ$ \\
\hline$G$ & Afra & Afrq & Afrq & Afra & Afrq & Afra & Afrq & NFra & NFrq & Afrq \\
\hline$G_{r}$ & ravr & ravr & ravv & ravr & ravv & rav & rav & rav & $q T \circ D$ & $q T \circ D$ \\
\hline$G_{r}$ & $991 \%$ & 9914 & 9914 & sqA4 & 9914 & $991 \%$ & gqAF & g9人t & fQSG & Y909 \\
\hline$Y_{1}$ & 9490 & 9990 & 9990 & 9990 & 9990 & 9990 & 9490 & 9990 & 9990 & 9990 \\
\hline$Y_{\mathrm{r}}$ & rrA。 & TrA。 & rYA。 & rrA。 & TrA。 & rYA。 & rrA。 & rrA。 & $\Delta Y_{0}$ & $\Delta r_{0}$ \\
\hline$Y_{r}$ & $\Delta \vee \varepsilon_{0}$ & $\Delta \vee \varphi_{0}$ & $\Delta V \varphi_{0}$ & $\Delta V \varphi_{0}$ & $\Delta V \varepsilon_{0}$ & $\Delta V \varepsilon_{0}$ & $\Delta \vee \varphi_{0}$ & $\Delta \vee \varphi_{0}$ & rAF。 & rAF。 \\
\hline$\mu_{\text {and }}$ & $0, \Delta v 1$ & $\circ, \Delta \wedge r$ & $0,9 \circ V$ & 0,919 & 0,841 & $0,84 r$ & 0,990 & 0,994 & $0,9 \vee 9$ & $0, v \Delta$ \\
\hline$\gamma$ & $0, \Delta V I$ & $0, \Delta V I$ & $0, \Delta v 1$ & $0, \Delta V I$ & $0, \Delta v 1$ & $0, \Delta V I$ & $0, \Delta V I$ & $0,0 v 1$ & $0, \Delta V I$ & $\circ$ \\
\hline$\mu_{1}$ & $0, \Delta V I$ & $0, \Delta V I$ & $0, \Delta V I$ & $0, \Delta V I$ & $0, \Delta V I$ & $0, \Delta V I$ & $0, \Delta V I$ & $0,0 v 1$ & $0,0 v 1$ & $\circ$ \\
\hline$\mu_{r}$ & 0,819 & 0,819 & 0,919 & 0,919 & 0,919 & 0,919 & 0,919 & 0,819 & 0,919 & 1 \\
\hline$\mu_{r}$ & $0,4 r q$ & $0,4 r q$ & $0,4 r q$ & $0,4 p q$ & $0,4 r q$ & $0,4 r q$ & $0,4 r q$ & $0, p r q$ & $0,4 Y q$ & 1 \\
\hline$\mu_{\psi}$ & $0, \Delta V I$ & $0, \Delta V I$ & $0, \Delta V I$ & $\circ, \Delta V I$ & $0, \Delta V I$ & $0, \Delta V I$ & $0, \Delta V I$ & $0, \Delta V I$ & $0, \Delta V I$ & 1 \\
\hline
\end{tabular}

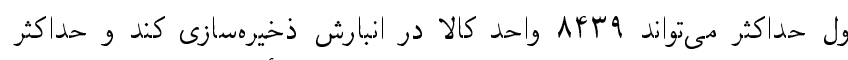

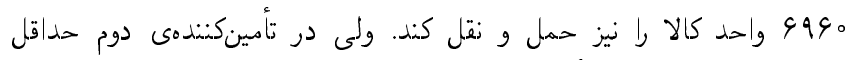

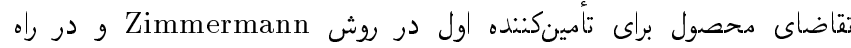

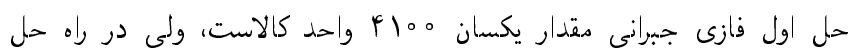

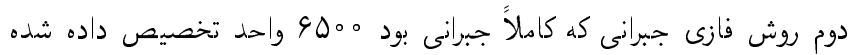

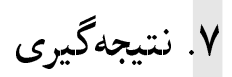

در اين نوشتار يك مدل جندهدفهى فازى براى مسئلى انتخاب تأمينكنده ارائه

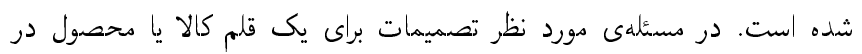

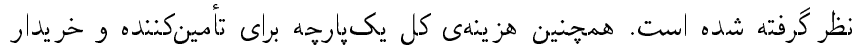

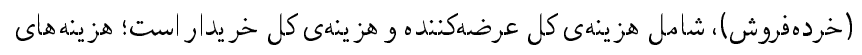

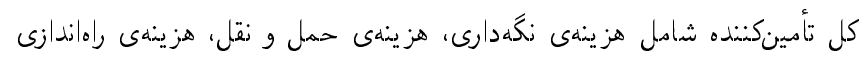

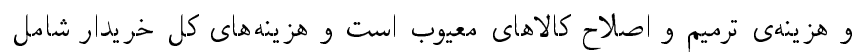

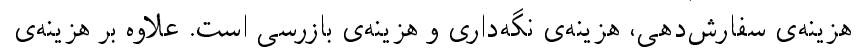

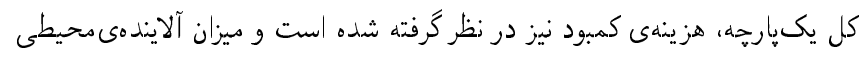

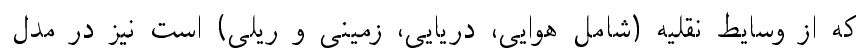

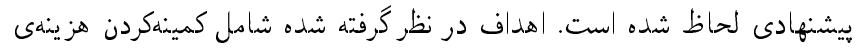

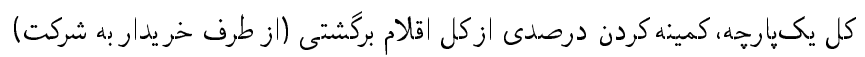

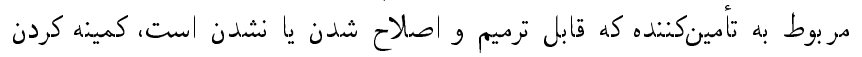

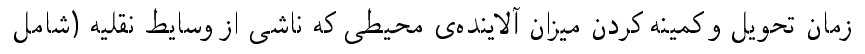

جدول 9. مقايسهى نتايج حاصل از حل مدل با استفاده از رويكرد فازى - Zimmer

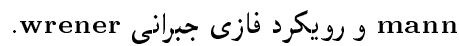

\begin{tabular}{|c|c|c|c|}
\hline \multicolumn{2}{|c|}{ روش فاز جبرانى Warner } & \multirow{2}{*}{$\begin{array}{c}\text { روش } \\
\text { Zimmermann }\end{array}$} & \multirow{2}{*}{ مقادير } \\
\hline راه حل دوم & راه حل اول & & \\
\hline$\wedge v_{0} \circ$ & $\wedge V_{0} \circ$ & $A V_{0} \circ$ & $X_{1}$ \\
\hline 4100 & 4100 & 4100 & $X_{r}$ \\
\hline$\psi \wedge 00$ & VYoo & VYoo & $X_{\Gamma}$ \\
\hline Aerq & AFra & Aerq & $G_{1}$ \\
\hline$q \mu .0$ & ravr & ravr & $G_{\mathrm{r}}$ \\
\hline 1909 & 99AF & $991 \mathrm{~F}$ & $G_{r}$ \\
\hline $999^{\circ}$ & $999^{\circ}$ & 9990 & $Y_{1}$ \\
\hline$\Delta Y_{0} \circ$ & rrA。 & rrA。 & $Y_{r}$ \\
\hline rAF。 & $\Delta \varphi \varphi_{0}$ & ovg. & $Y_{\tau}$ \\
\hline
\end{tabular}

لازم به توضيح است كه روش فازى جبرانى كه قبلاً حل شده داراى دو راه

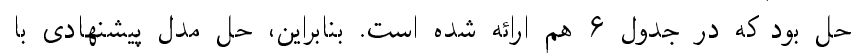

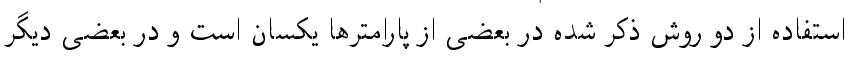

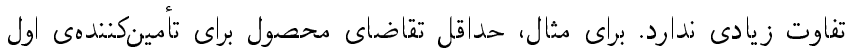

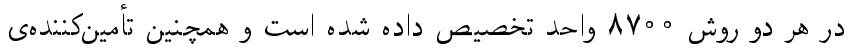




$$
\begin{aligned}
& \text { - در نظر گرفتن هارامترهايى هميجون سطح خدمترسانى، نرخ قابليت اطمينان }
\end{aligned}
$$

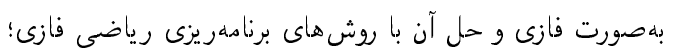

$$
\begin{aligned}
& \text { ــ در نظر گرفتن هسيرهاى حمل و نقل وسايط نقليه (زمينى، ريلى، هوايى و }
\end{aligned}
$$

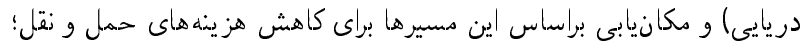

$$
\begin{aligned}
& \text { - طرحريزى كارخانها براى تسهيل و كاهش هزينها و كاهش آلايندهايى كه از }
\end{aligned}
$$

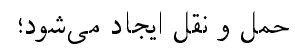

$$
\begin{aligned}
& \text { - استفاده از الكوريتمهاى فراابتكارى براى حل مدلهاى جندهدفه و مقايسه با }
\end{aligned}
$$

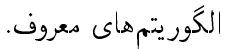

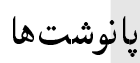

1. lead time

2. effective cost

\section{منابع (References)}

1. Aissaoui, N., Haouari, M. and Hassini, E. "Supplier selection and order lot sizing modeling: Areview", Computers \& Operations Research, 34, pp. 3516-3540 (2007).

2. Ghodsypour, S.H. and O'Brien, C. "A decision support system for supplier selection using an integrated analytic hierarchy process and linear programming", International Journal of Production Economics, 56-57(1-3), pp. 199-212 (1998).

3. Weber, C.A., Current, J.R. and Benton, W.C. "Vendor selection criteria and methods", EuropeanJournal of $\mathrm{Op}$ erational Research, 50(1), pp. 2-18 (2000).

4. Dahel, N.E. "Vendor selection and order quantity allocation in volume discount environments", Supply Chain Management: An International Journal, 8(4), pp. 335$342(2003)$.

5. Kumar, M., Vart, P. and Shankar, R. "A fuzzy goal programming approach for supplier selectionproblem in a supply chain", Computer and Industrial Engineering, 46, pp. 69-85 (2004).

6. Amid, A., Ghodsypour, S.H. and O'Brien, Ch. "Fuzzy multi-objective linear model for supplierselection in a supply chain", International Journal of Production Economics, 104, pp. 394-407 (2006).

7. Amid, A., Ghodsypour, S.H. and O'Brien, Ch. "A weighted additive fuzzy multi-objective model forthe supplier selection problem under price breaks in a supply chain", International Journal of Production Economics, 121, pp. 323-332 (2009).

8. Ghodsypour, S.H. and O'Brien, Ch. "A weighted maxmin model for fuzzy-objective supplier selection in a
هوايى، دريايى، زمينى و ريلى) هستند. براى حل مدل بيشنهادى از رويكردهاى

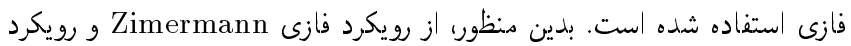
فازى جبرانى Werner استفاده شده است و در انتها اين دو روش با هم دقايسه شدهاند.

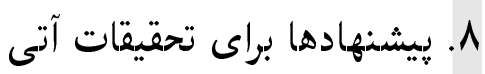

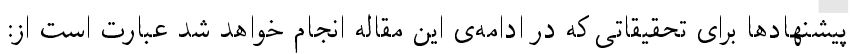

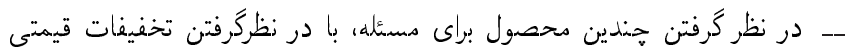
براى مسئله؛

supply chain", International Journal of Production Economics, 131(2011), pp. 139-145 (2010).

9. Yücel, A. and Güneri, A.F. "A weighted addittive programming approach for multi-criteria supplier selection", Expert Systems with Applications, 38(2011), pp. 6281-6286 (2010).

10. Shaw, K., Shankar, R., Yadav, S.S., and Thakur, S.L. "Supplier selection using fuzzy AHP and fuzzymultiobjective linear programming for developing low carbon supply chain", Expert Systems with Applications, 39(9), pp. 8182-8192 (2012).

11. Paksoy, T., Pehlivan, Y.N. and Özceylan, E. "Application of fuzzy optimization to a supply chain network design: A case study of an edible vegetable oils manufacturer", Applied Mathematical Modelling, 36(6), pp. 2762-2776 (2012).

12. Zimmermann, H.J., Fuzzy Set Theory and its Applications, (2nd Revised ed.), Boston, Dordrecht, London: Kluwer Academic Publishers (6th printing) (1993).

13. Lee, E.S. and Li, R.J. "Fuzzy multiple objective programming and compromise programming withPareto optimum", Fuzzy Sets and Systems, 53, pp. 275-288 (1993).

14. Guu, S.M. and Wu, Y.K. "Weighted coefficients in twophase approach for solving the multipleobjective programming problems", Fuzzy Sets and Systems, 85, pp. 45-48 (1997).

15. Lee, E-K., Ha, S. and Kim, S.-K. "Supplier selection and management system considerin relationships in supply chain management", IEEE Transactions on Engineering Management, 48(3), pp. 307-318 (2001).

16. Ozkok, B.A. and Tiryaki, F. "A compensatory fuzzy approach to multi-objective linear supplierselection problem with multiple-item", Expert Systems with Applications, 38, pp. 11363-11368 (2011).

17. Werners, B.M. "Aggregation models in mathematical programming", In G. Mitra (Ed.), Mathematical Models for Decision Support, Berlin, Springer, pp. 295-305 (1988). 